\title{
Relation between reperfusion and hemorrhagic transformation in acute ischemic stroke
}

\author{
Alexander D. Horsch ${ }^{1,2}$ - Jan Willem Dankbaar ${ }^{1}$ - Yolanda van der Graaf ${ }^{3}$. \\ Joris M. Niesten ${ }^{1} \cdot$ Tom van Seeters $^{1} \cdot$ Irene C. van der Schaaf $^{1} \cdot$ L. Jaap Kappelle ${ }^{4}$. \\ Birgitta K. Velthuis ${ }^{1}$ • On behalf of the DUST investigators
}

Received: 7 July 2015 / Accepted: 10 August 2015 / Published online: 4 September 2015

(C) The Author(s) 2015. This article is published with open access at Springerlink.com

\begin{abstract}
Introduction Intravenous recombinant tissue plasminogen activator (IV-rtPA) is given in acute ischemic stroke patients to achieve reperfusion. Hemorrhagic transformation (HT) is a serious complication of IV-rtPA treatment and related to blood-brain barrier (BBB) injury. It is unclear whether HT occurs secondary to reperfusion in combination with ischemic BBB injury or is caused by the negative effect of IV-rtPA on BBB integrity. The aim of this study was to establish the association between reperfusion and the occurrence of HT. Methods From the DUST study, patients were selected with admission and follow-up non-contrast CT (NCCT) and CT perfusion (CTP) imaging, and a perfusion deficit in the middle cerebral artery territory on admission. Reperfusion was categorized qualitatively as reperfusion or no-reperfusion by visual comparison of admission and follow-up CTP. Occurrence of HT was assessed on follow-up NCCT. The association between reperfusion and occurrence of HT on follow-up was estimated by calculating odds ratios (ORs) and $95 \%$ confidence intervals (CIs) with additional stratification for IV-rtPA treatment.

Results Inclusion criteria were met in 299 patients. There was no significant association between reperfusion and HT (OR
\end{abstract}

Alexander D. Horsch

alexanderhorsch@gmail.com

1 Department of Radiology, University Medical Center Utrecht, Heidelberglaan 100, HP E01.132, 3584 CX Utrecht, The Netherlands

2 Department of Radiology, Rijnstate Hospital, Arnhem, The Netherlands

3 Julius Center for Health Sciences and Primary Care, Utrecht, The Netherlands

4 Department of Neurology, Utrecht Stroke Center, University Medical Center, Utrecht, The Netherlands
$1.295 \%$ CI 0.5-3.1). In patients treated with IV-rtPA $(n=203)$, the OR was $1.3(95 \%$ CI $0.4-4.0)$, and in patients not treated with IV-rtPA $(n=96)$, the OR was $0.8(95 \%$ CI $0.1-4.5)$. HT occurred in $14 \%$ of the IV-rtPA patients and in $7 \%$ of patients without IV-rtPA ( $95 \% \mathrm{CI}$ of difference -1 to $14 \%$ ).

Conclusion Our results suggest that the increased risk of HT after acute ischemic stroke treatment is not dependent on the reperfusion status.

Keywords Ischemic stroke $\cdot \mathrm{CT}$ perfusion $\cdot \mathrm{rtPA}$.

Reperfusion $\cdot$ Hemorrhagic transformation

\section{Introduction}

Timely restoration of the downstream capillary blood flow (reperfusion) by recanalization of the occluded vessel in acute ischemic stroke patients is associated with favorable clinical outcome $[1,2]$. However, reperfusion has also been associated with the occurrence of hemorrhagic transformation (HT) through a mechanism called reperfusion injury [1, 3-5]. HT incorporates all types of post-ischemic hemorrhages, ranging from the smaller hemorrhagic infarction (HI) type 1 or 2, to the larger parenchymal hemorrhage (PH) type 1 or 2. Especially, the PH types may increase the risk of worse clinical outcome [6-9]. To induce reperfusion and thereby improve clinical outcome, intravenous recombinant tissue plasminogen activator (IV-rtPA) can be given within $4.5 \mathrm{~h}$ after symptom onset [10]. However, IV-rtPA also increases the risk of HT by its thrombolytic action as well as by causing direct damage to the blood-brain barrier (BBB) [10-13]. It is unclear whether HT after ischemic stroke is caused mainly by reperfusion of an ischemic area with damage to the BBB or by the detrimental effects of IV-rtPA on the BBB [3,9]. To further 
explore this topic, first the relation between reperfusion and the occurrence of HT needs to be investigated.

The purpose of this study was to investigate the association between reperfusion and the occurrence of HT, both in patients treated with and without IV-rtPA.

\section{Materials and methods}

\section{Study design}

The Dutch acute stroke study (DUST) is a large prospective multicenter cohort study that aims to assess the value of $\mathrm{CT}$ perfusion (CTP) and CT angiography (CTA) in addition to patient characteristics and non-contrast $\mathrm{CT}$ (NCCT) for prediction of outcome in patients with acute ischemic stroke (ClinicalTrials.gov NCT00880113). Patients were included in 14 hospitals between May 2009 and August 2013 [14].

Inclusion criteria for the DUST were as follows: age $>18$ years, suspected ischemic stroke of less than $9 \mathrm{~h}$ in duration, and National Institutes of Health Stroke Scale (NIHSS) $\geq 2$, or 1 if an indication for IV-rtPA was present. Exclusion criteria were known renal failure, contraindications for iodinated contrast material, and hemorrhage or another diagnosis seen on NCCT to explain the stroke symptoms. This study was approved by the central medical ethics committee in the University Medical Center (UMC) Utrecht and the local institutional ethical review boards of the participating hospitals. Patients or family gave signed informed consent unless a patient died before consent could be obtained, in which case the need for consent was waived by the medical ethics committee [14].

\section{Patient selection}

From the prospectively collected DUST database, patients were retrospectively selected with (1) admission and followup NCCT and CT perfusion and (2) a perfusion deficit in the middle cerebral artery territory on admission CTP. Exclusion criteria were as follows: (1) intra-arterial treatment, (2) poor quality CTP, or (3) absence of one of the Alberta Stroke Program Early CT Score (ASPECTS) levels on CTP. The inclusion process is clarified in the flow chart (Fig. 1). Collected clinical data were age, sex, history of stroke or hypertension, NIHSS on admission, IV-rtPA treatment, and time from symptom onset to admission CT scan series.

\section{Imaging protocol and post-processing}

In all DUST patients, NCCT, CTP, and CTA were performed on admission. Non-obligatory follow-up NCCT was planned after 3 days ( \pm 2 days) and also performed in case of clinical deterioration. Additional follow-up CTA and CTP were performed if possible. For the current study, patients were included only if

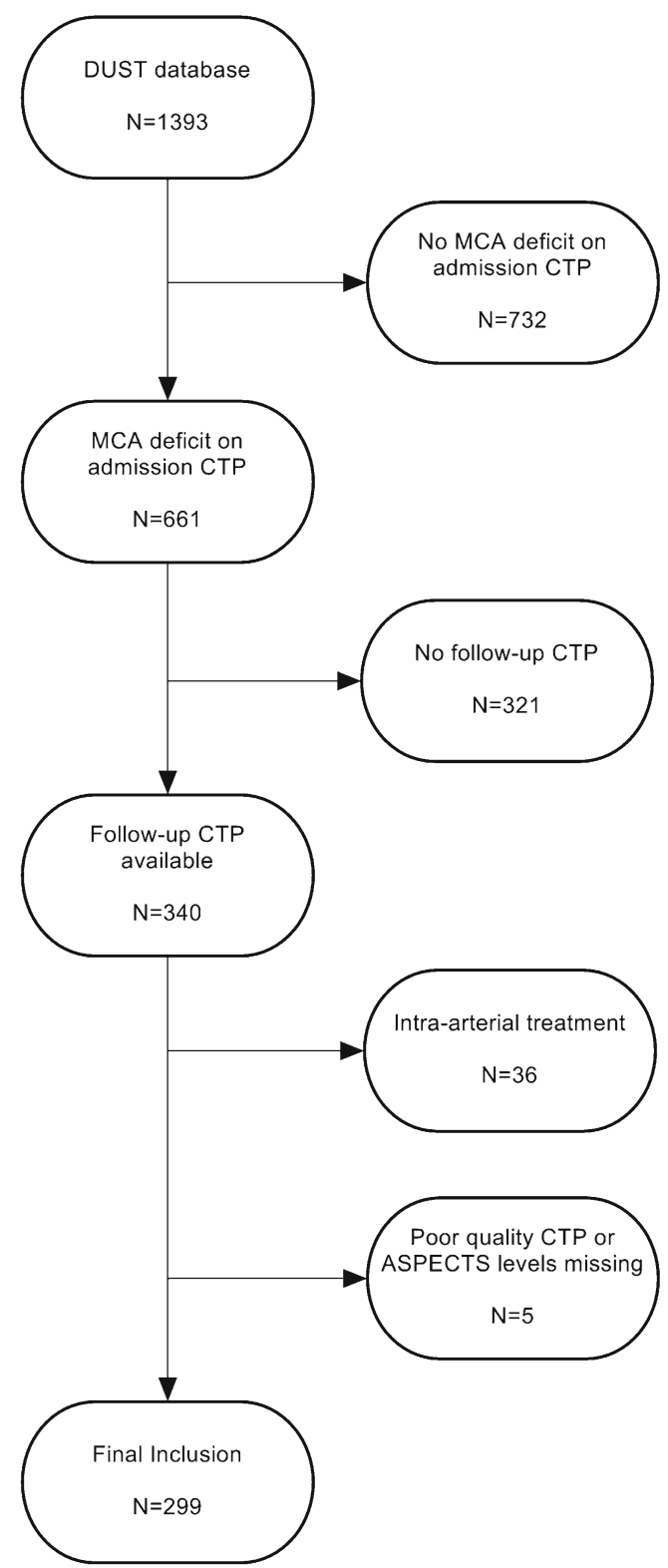

Fig. 1 Flow chart patient selection

follow-up imaging was performed with NCCT and CTP. All imaging studies were performed on multidetector CT scanners ranging from 40 to 320 detectors. The CT protocol has been described previously [14].

In short, the CTP involved successive gantry rotations in cine mode during intravenous administration of iodinated contrast material ( $40 \mathrm{ml}$ non-ionic contrast) followed by $40 \mathrm{ml}$ of saline, both with a flow of $6 \mathrm{ml} / \mathrm{s}$. The CTP covered at least the level of the basal ganglia to the lateral ventricles to be able to assess ASPECTS levels 1 and 2, and compare between scanners with different number of detectors [15].

From the acquired CTP data, color maps were created for cerebral blood volume (CBV), cerebral blood flow (CBF), mean transit time (MTT), and time to peak (TTP) utilizing 
commercially available CTP software (Extended Brilliance workstation 4.5, Philips Healthcare). This software uses a deconvolution-based method which determines the MTT by the difference in first moment of tissue and arterial time attenuation curves $[16,17]$. To calculate the CBF from the MTT, the software applies the central volume principle which is the most accurate for low injection rates of iodinated contrast material [18]. The internal carotid artery (if available in the scan range) or anterior cerebral artery was chosen as arterial input function [19]. The superior sagittal sinus was used as venous output function.

All data and imaging processing was done centrally in the UMC Utrecht. Scans were evaluated by one of three observers with more than 5 years of stroke imaging experience (B.K.V., I.C.vd.S, J.W.D.). The side of symptoms was provided, but observers were blinded to other clinical and imaging data. Consensus was reached for ambiguous findings by an extra review by two of the three radiologists, also blinded to other clinical and imaging data. The number of consensus agreement cases was not collected.

\section{Reperfusion}

Reperfusion status was analyzed by visual comparison of the size of the perfusion abnormality on admission and follow-up CTP maps for CBV, CBF, MTT, and TTP. Reperfusion was classified qualitatively in a reperfusion and a no-reperfusion group. No-reperfusion was defined as the absence of any visually apparent change in the size of the perfusion deficit on follow-up CTP compared to the admission CTP. Partial reperfusion and hyperperfusion were included in the reperfusion group and an enlarged or new perfusion deficit in the noreperfusion group.

\section{Hemorrhagic transformation}

The follow-up NCCT was evaluated for the presence of hemorrhage. Hemorrhages were classified according to the European Cooperative Acute Stroke Study (ECASS)-1 criteria: HI1 (small petechiae along the margins of infarct), HI-2 (confluent petechiae within infarcted area but no space-occupying effect), PH-1 (blood clots in $30 \%$ or less of the infarcted area with some slight space-occupying effect), and PH-2 (blood clots of more than $30 \%$ of infarcted area with substantial space-occupying effect) $[20,7]$.

\section{Statistical analysis}

Patient characteristics were presented as number and percentages, mean and standard deviation (SD), or median and interquartile range (IQ). Differences between patients with and without IV-rtPA treatment were tested with the $\chi^{2}$ test to compare categorical variables and the Mann-Whitney $U$ test for continuous variables.

The primary outcome was occurrence of any HT (all ECASS categories together). We estimated the association between reperfusion and the occurrence of HT by calculating odds ratios with $95 \%$ confidence intervals (95\%CIs) in the total group of patients and in the sub-groups with and without IV-rtPA treatment.

\section{Results}

From the DUST database of 1393 patients with complete admission data, 299 patients met inclusion criteria for this study. A total of 36 HTs $(12 \%)$ occurred in this selected patient group (ECASS HI-1, 13; HI-2, 15; PH-1, 7; and PH-2, 1). The overall percentage was comparable to the percentage of HT in the whole DUST database $(11 \%)$. The main reasons for exclusion was the absence of an ischemic deficit on admission CTP $(n=732)$ or because no follow-up CTP imaging was done $(n=321)$. No significant difference was found in 3-month mRS between included and all excluded patients.

Clinical and imaging characteristics are summarized in Table 1. Treatment with IV-rtPA was given in 203 of the 299 patients $(68 \%)$. Patients treated with IV-rtPA less often had a prior history of stroke or hypertension, had a significantly higher median NIHSS on admission ( 9 vs 6), and had a shorter median time to scan than patients who did not receive IV-rtPA (84 vs $218 \mathrm{~min})$.

Reperfusion (median assessed at day 3) was visible in 241 patients $(81 \%)$. There was no significant association between HT and reperfusion status in all patients (odds ratio (OR) 1.2, 95\%CI 0.5-3.1). Of the 36 (12\%) patients with HT, 29 received IV-rtPA treatment and 7 did not. HT occurred twice as often in patients treated with IV-rtPA compared to patients not treated with IV-rtPA (14 vs $7 \%$ with a $95 \% \mathrm{CI}-1$ to $14 \%$ for the difference). Both in patients treated with IV-rtPA and in patients not treated with IV-rtPA, there was no significant association between reperfusion and HT (OR 1.3 (95\% CI 0.4-4.0) and OR 0.8 (95\%CI 0.1-4.5), respectively).

The results of a sub-analysis show that of the 28 patients with HI-type hemorrhages, 25 (89\%) showed reperfusion, while 3 (11\%) did not. Of the eight patients with PH type hemorrhage, five $(63 \%)$ showed reperfusion while three (37\%) did not.

\section{Discussion}

The main finding in this study is that the overall occurrence of hemorrhagic transformation 3 days after onset of acute ischemic stroke does not seem to be associated with reperfusion.

Our findings are in contradiction to Fiehler et al. 2005 who suggested, in a retrospective MRI study in which HT occurred 
Table 1 Baseline clinical and imaging characteristics

\begin{tabular}{|c|c|c|c|c|}
\hline & $\begin{array}{l}\text { All patients } \\
N=299\end{array}$ & $\begin{array}{l}\mathrm{rtPA} \\
N=203\end{array}$ & $\begin{array}{l}\text { No rtPA } \\
N=96\end{array}$ & $P$ value \\
\hline \multicolumn{5}{|l|}{ Clinical parameters } \\
\hline Age, median (IQ) & $68(58-77)$ & $68(56-75)$ & $70(61-78)$ & 0.10 \\
\hline Female sex, $n(\%)$ & $116(39)$ & $79(39)$ & $37(39)$ & 0.95 \\
\hline Prior stroke, $n(\%)$ & $68(23)$ & $35(17)$ & $33(34)$ & $0.001 *$ \\
\hline Hypertension, $n(\%)$ & $154(52)$ & $95(47)$ & $59(62)$ & $0.02 *$ \\
\hline NIHSS, median (IQ) & $8(5-14)$ & $9(6-15)$ & $6(3-12)$ & $0.002 *$ \\
\hline \multicolumn{5}{|l|}{ Imaging parameters } \\
\hline Time to admission scan, minutes, median (IQ) & $103(65-163)$ & $84(60-133)$ & $218(133-315)$ & $0.006^{*}$ \\
\hline \multicolumn{5}{|l|}{ NCCT } \\
\hline $\mathrm{HT}, n(\%)$ & $36(12)$ & $29(14)$ & $7(7)$ & 0.08 \\
\hline \multicolumn{5}{|l|}{ СТP } \\
\hline No reperfusion, $n(\%)$ & $58(19)$ & $34(17)$ & $24(25)$ & 0.09 \\
\hline
\end{tabular}

$\chi^{2}$ test was used to compare categorical variables and Mann-Whitney $U$ test for continuous variables

CTP CT perfusion, HT hemorrhagic transformation, IQ interquartile range, NCCT non-contrast CT, NIHSS National Institutes of Health Stroke Scale, $r t P A$ recombinant tissue plasminogen activator

$* P<0.05$

in 19 of the 51 patients, that HT in patients treated with IVrtPA might be caused by a higher incidence of local reperfusion in the HT area [3]. However, in this study, no significant difference in the occurrence of reperfusion between patients with and without HT could be shown if the entire admission perfusion abnormality area was considered. Moreover, the definition of reperfusion used in that study was only based on changes in TTP delay measured with MRI instead of CT, which could lead to differences in measuring the infarct core and penumbra and hence to different results [3]. Another retrospective MRI study, with HT occurring in 22 of the 144 patients, stated that reperfusion was the most significant independent predictor of early BBB disruption and that this BBB disruption was an independent predictor of HT [21]. However, they did not show a direct relation between reperfusion and HT. BBB disruption was defined as post-gadolinium CSF enhancement, a technique not frequently used in clinical practice. Moreover, only $25 \%$ of their patients received IV-rtPA, and no significant association between IV-rtPA and HT was shown [21].

To our knowledge, our study is the largest prospectively collected dataset to evaluate the association between reperfusion and HT. The overall percentage of HT patients in our study was within the range of previously published data on CT follow-up literature $[12,22]$. There was a clear difference in the occurrence of HT between patients treated with IV-rtPA and patients not treated with IV-rtPA, despite the absence of an association with reperfusion. This might be an indication that the delivery of rtPA to the ischemic area and not the reperfusion itself results in HT. However, other factors like stroke severity or time to treatment may also play a role.
Treatment with IV-rtPA was given in $68 \%$ of our patients. This percentage is much higher compared to that of other studies and possibly reflects the increased stroke awareness in The Netherlands and subsequent shorter time to admission. A selection bias in the DUST study with preference given to inclusion of patients eligible for IV-rtPA treatment could also be the reason for this higher percentage of treated patients.

The results of the sub-analysis of the HI-type and PH-type hemorrhages suggest that there is a trend toward a higher perfusion rate in patients developing HI-type hemorrhages, while PH-type hemorrhages seem less related to reperfusion. This is in accordance with the findings of ECASS-2 which showed better outcomes with HI-type hemorrhage and worse outcomes with PH-type hemorrhages, possibly related to reperfusion status [23]. Unfortunately, the numbers in the subanalysis are too low to perform meaningful statistical analysis on these sub-groups. As we already derived our population from the whole DUST database, we were unable to test this in a larger cohort of patients.

Although we did not show a relation between reperfusion and the occurrence of hemorrhagic transformation, it is possible that this is due to the fact that the exact location of the hemorrhage is difficult to ascertain and that the reperfusion in a very focal area could be of importance. It could be argued that with the use of higher-resolution thin-sliced CTP and added filtering and noise reduction, this relationship could be better determined in future studies [24].

This study has some limitations. First, although the overall number of hemorrhages was comparable to previously published data, the number of symptomatic hemorrhages (defined as $\mathrm{PH}-2$ ) in this series is low compared to that in some other 
studies $[25,22]$. Most symptomatic hemorrhages $(n=23)$ are not included because the inclusion criteria for this study required a CTP at follow-up. Patients with symptomatic HT may have been too agitated or hemodynamically unstable to lie still long enough to perform this follow-up CTP, and many of the PH-2 outcomes from the DUST database could not be included in this analysis because follow-up CTP was missing. Another explanation for the low number of PH-2's may be the relatively short time to treatment in our study. It is known that longer time to treatment is related to occurrence of HT [25]. We included smaller hemorrhages (HI-1 and HI-2) in our analysis since they are also related to poor outcome in larger studies [26-28]. Nevertheless, due to the low number of $\mathrm{PH}-2$, our results must be interpreted with caution and are mainly applicable for populations with smaller hemorrhages.

Second, the timing of most follow-up scans was around 3 days. Reperfusion and recanalization are known to continue up to several weeks but the "time is brain" concept states that reperfusion is only relevant if it occurs within several hours after onset of ischemia [29]. It could be argued that reperfusion measurement at an earlier time point would be more appropriate. But, for practical purposes and to minimize discomfort for participating patients, we had to compromise between timing of HT detection and early reperfusion assessment. Moreover, because patients did receive an additional NCCT in case of clinical deterioration after the scheduled follow-up scans were made, it is unlikely that significant hemorrhages were missed.

Third, we excluded patients with intra-arterial treatment as most of these patients were treated with a combination of mechanical thrombectomy and intra-arterial thrombolysis, which probably has an added but unknown effect on the integrity of the $\mathrm{BBB}$.

Fourth, reperfusion status was not quantitatively assessed but dichotomized as reperfusion or no-reperfusion by visual comparison of the admission and follow-up CTP images. Little is known about the quantitative assessment of reperfusion with CTP, and no universal thresholds for the assessment of change of CTP deficits are available. Moreover, the discrepancy rate for quantitative assessment of reperfusion with the TIMI reperfusion score on angiography has been described to be as high as $41 \%$, which seems to justify using a simple qualitative assessment [30]. Qualitative interpretation of CTP has shown good to excellent agreement rates between observers [31-33].

\section{Conclusion}

The occurrence of hemorrhagic transformation does not seem to be associated with reperfusion. This suggests that other causes, like ischemic injury or the effects of IV-rtPA, are more important in the occurrence of hemorrhagic transformation in acute ischemic stroke.
Acknowledgments This study was supported by grants from The Netherlands Heart Foundation (grant numbers 2008 T034 and 2012 T061) and the Nuts Ohra Foundation (grant number 0903-012). We thank the Dutch acute stroke study (DUST) investigators: Academic Medical Center, Amsterdam, The Netherlands (Majoie CB, Roos YB); Catharina Hospital, Eindhoven, The Netherlands (Duijm LE, Keizer K); Erasmus Medical Center, Rotterdam, The Netherlands (van der Lugt A, Dippel DW); Gelre Hospitals, Apeldoorn, The Netherlands (Droogh - de Greve KE, Bienfait HP); Leiden University Medical Center, Leiden, The Netherlands (van Walderveen MA, Wermer MJ); Medical Center Haaglanden, The Hague, The Netherlands (Lycklama à Nijeholt GJ, Boiten J); Onze Lieve Vrouwe Gasthuis, Amsterdam, The Netherlands (Duyndam D, Kwa VI); Radboud University Nijmegen Medical Centre, Nijmegen, The Netherlands (Meijer FJ, van Dijk EJ); Rijnstate Hospital, Arnhem, The Netherlands (Kesselring FO, Hofmeijer J); St. Antonius Hospital, Nieuwegein, The Netherlands (Vos JA, Schonewille WJ); St. Elisabeth Hospital, Tilburg, The Netherlands (van Rooij WJ, de Kort PL); St. Franciscus Hospital, Rotterdam, The Netherlands (Pleiter CC, Bakker SL); VU Medical Center, Amsterdam, The Netherlands (Bot J, Visser MC); and University Medical Center Utrecht, Utrecht, The Netherlands (Velthuis BK, van der Schaaf IC, Dankbaar JW, Mali WP, van Seeters T, Horsch AD, Niesten JM, Biessels GJ, Kappelle LJ, Luitse MJ, van der Graaf Y).

Ethical standards and patient consent We declare that all human and animal studies have been approved by the Medisch Ethische Toetsingscommissie UMC Utrecht and have therefore been performed in accordance with the ethical standards laid down in the 1964 Declaration of Helsinki and its later amendments. We declare that all patients or family gave informed consent prior to inclusion in this study, unless a patient died before consent could be obtained, in which case the medical ethics committee waived consent.

Conflict of interest BKV is a regular presenter for Philips Healthcare.

Open Access This article is distributed under the terms of the Creative Commons Attribution 4.0 International License (http://creativecommons.org/ licenses/by/4.0/), which permits unrestricted use, distribution, and reproduction in any medium, provided you give appropriate credit to the original author(s) and the source, provide a link to the Creative Commons license, and indicate if changes were made.

\section{References}

1. Albers GW, Thijs VN, Wechsler L, Kemp S, Schlaug G, Skalabrin E, Bammer R, Kakuda W, Lansberg MG, Shuaib A, Coplin W, Hamilton S, Moseley M, Marks MP (2006) Magnetic resonance imaging profiles predict clinical response to early reperfusion: the diffusion and perfusion imaging evaluation for understanding stroke evolution (DEFUSE) study. Ann Neurol 60(5):508-517. doi:10.1002/ana.20976

2. Soares BP, Tong E, Hom J, Cheng SC, Bredno J, Boussel L, Smith WS, Wintermark M (2010) Reperfusion is a more accurate predictor of follow-up infarct volume than recanalization: a proof of concept using CT in acute ischemic stroke patients. Stroke 41:e34-40. doi:10.1161/STROKEAHA.109.568766

3. Fiehler J, Remmele C, Kucinski T, Rosenkranz M, Thomalla G, Weiller C, Zeumer H, Rother J (2005) Reperfusion after severe local perfusion deficit precedes hemorrhagic transformation: an MRI study in acute stroke patients. Cerebrovasc Dis 19(2):117124. doi: $10.1159 / 000083180$

4. Warach S (2004) Evidence of reperfusion injury, exacerbated by thrombolytic therapy, in human focal brain ischemia using a novel 
imaging marker of early blood-brain barrier disruption. Stroke 35(suppl I):2659-2661. doi:10.1161/01.str.0000144051.32131.09

5. Molina CA, Alvarez-Sabin J (2009) Recanalization and reperfusion therapies for acute ischemic stroke. Cerebrovasc Dis 27(Suppl 1): 162-167. doi:10.1159/000200455

6. Rudolf J, Grond M, Stenzel C, Neveling M, Heiss WD (1998) Incidence of space-occupying brain edema following systemic thrombolysis of acute supratentorial ischemia. Cerebrovasc Dis 8(3):166-171. doi:10.1159/000015843

7. Berger C, Fiorelli M, Steiner T, Schabitz WR, Bozzao L, Bluhmki E, Hacke W, von Kummer R (2001) Hemorrhagic transformation of ischemic brain tissue: asymptomatic or symptomatic? Stroke 32(6): 1330-1335. doi:10.1161/01.STR.32.6.1330

8. Molina CA, Alvarez-Sabin J, Montaner J, Abilleira S, Arenillas JF, Coscojuela P, Romero F, Codina A (2002) Thrombolysis-related hemorrhagic infarction: a marker of early reperfusion, reduced infarct size, and improved outcome in patients with proximal middle cerebral artery occlusion. Stroke 33(6):1551-1556. doi:10.1161/01. STR.0000016323.13456.E5

9. Khatri P, Wechsler LR, Broderick JP (2007) Intracranial hemorrhage associated with revascularization therapies. Stroke 38(2): 431-440. doi:10.1161/01.STR.0000254524.23708.c9

10. Lees KR, Bluhmki E, von Kummer R, Brott TG, Toni D, Grotta JC, Albers GW, Kaste M, Marler JR, Hamilton SA, Tilley BC, Davis SM, Donnan GA, Hacke W, Allen K, Mau J, Meier D, del Zoppo G, De Silva DA, Butcher KS, Parsons MW, Barber PA, Levi C, Bladin C, Byrnes G (2010) Time to treatment with intravenous alteplase and outcome in stroke: an updated pooled analysis of ECASS, ATLANTIS, NINDS, and EPITHET trials. Lancet 375(9727): 1695-1703. doi:10.1016/S0140-6736(10)60491-6

11. Kassner A, Roberts TPL, Moran B, Silver FL, Mikulis DJ (2009) recombinant tissue plasminogen activator increases blood-brain barrier disruption in acute ischemic stroke: an MR imaging permeability study. AJNR Am J Neuroradiol 30(10):1864-1869. doi:10. 3174/ajnr.A1774

12. NINDS rssg (1995) Tissue plasminogen activator for acute ischemic stroke. The National Institute of Neurological Disorders and Stroke rt-PA Stroke Study Group. N Engl J Med 333(24):15811587. doi:10.1056/NEJM199512143332401

13. Saqqur M, Tsivgoulis G, Molina CA, Demchuk AM, Siddiqui M, Alvarez-Sabin J, Uchino K, Calleja S, Alexandrov AV (2008) Symptomatic intracerebral hemorrhage and recanalization after IV rt-PA: a multicenter study. Neurology 71(17):1304-1312. doi:10. 1212/01.wnl.0000313936.15842.0d

14. van Seeters T, Biessels GJ, van der Schaaf IC, Dankbaar JW, Horsch AD, Luitse MJ, Niesten JM, Mali WP, Kappelle LJ, van der Graaf Y, Velthuis BK (2014) Prediction of outcome in patients with suspected acute ischaemic stroke with CT perfusion and CT angiography: the Dutch acute stroke trial (DUST) study protocol. BMC Neurol 14:37. doi:10.1186/1471-2377-14-37

15. Barber PA, Demchuk AM, Zhang J, Buchan AM (2000) Validity and reliability of a quantitative computed tomography score in predicting outcome of hyperacute stroke before thrombolytic therapy. Lancet 355(9216):1670-1674. doi:10.1016/s0140-6736(00) 02237-6

16. Ostergaard L, Weisskoff RM, Chesler DA, Gyldensted C, Rosen BR (1996) High resolution measurement of cerebral blood flow using intravascular tracer bolus passages. Part I: Mathematical approach and statistical analysis. Magn Reson Med 36(5):715-725. doi:10.1002/mrm.1910360510

17. Axel L (1981) A method of calculating brain blood flow with a CT dynamic scanner. Adv Neurol 30:67-71

18. Wintermark M, Maeder P, Thiran JP, Schnyder P, Meuli R (2001) Quantitative assessment of regional cerebral blood flows by perfusion CT studies at low injection rates: a critical review of the underlying theoretical models. Eur Radiol 11(7):1220-1230. doi: 10.1007/s003300000707

19. Niesten JM, van der Schaaf IC, Riordan AJ, de Jong HW, Mali WP, Velthuis BK (2013) Optimisation of vascular input and output functions in CT-perfusion imaging using 256(or more)-slice multidetector CT. Eur Radiol 23(5):1242-1249. doi:10.1007/s00330-0122731-8

20. Hacke W, Kaste M, Fieschi C, Toni D, Lesaffre E, von Kummer R, Boysen G, Bluhmki E, Hoxter G, Mahagne MH et al (1995) Intravenous thrombolysis with recombinant tissue plasminogen activator for acute hemispheric stroke. The European Cooperative Acute Stroke Study (ECASS). JAMA 274(13):1017-1025. doi: 10.1001/jama.1995.03530130023023

21. Latour LL, Kang DW, Ezzeddine MA, Chalela JA, Warach S (2004) Early blood-brain barrier disruption in human focal brain ischemia. Ann Neurol 56(4):468-477. doi:10.1002/ana.20199

22. Wahlgren N, Ahmed N, Davalos A, Ford GA, Grond M, Hacke W, Hennerici MG, Kaste M, Kuelkens S, Larrue V, Lees KR, Roine RO, Soinne L, Toni D, Vanhooren G (2007) Thrombolysis with alteplase for acute ischaemic stroke in the Safe Implementation of Thrombolysis in Stroke-Monitoring Study (SITS-MOST): an observational study. Lancet 369(9558):275-282. doi:10.1016/S01406736(07)60149-4

23. Hacke W, Kaste M, Fieschi C, von Kummer R, Davalos A, Meier D, Larrue V, Bluhmki E, Davis S, Donnan G, Schneider D, DiezTejedor E, Trouillas P (1998) Randomised double-blind placebocontrolled trial of thrombolytic therapy with intravenous alteplase in acute ischaemic stroke (ECASS II). Lancet 352(9136):12451251. doi:10.1016/s0140-6736(98)08020-9

24. Mendrik AM, Vonken EJ, van Ginneken B, de Jong HW, Riordan A, van Seeters T, Smit EJ, Viergever MA, Prokop M (2011) TIPS bilateral noise reduction in 4D CT perfusion scans produces highquality cerebral blood flow maps. Phys Med Biol 56(13):38573872. doi:10.1088/0031-9155/56/13/008

25. Hacke W, Donnan G, Fieschi C, Kaste M, von Kummer R, Broderick JP, Brott T, Frankel M, Grotta JC, Haley EC Jr, Kwiatkowski T, Levine SR, Lewandowski C, Lu M, Lyden P, Marler JR, Patel S, Tilley BC, Albers G, Bluhmki E, Wilhelm M, Hamilton S (2004) Association of outcome with early stroke treatment: pooled analysis of ATLANTIS, ECASS, and NINDS rt-PA stroke trials. Lancet 363(9411):768-774. doi:10.1016/S01406736(04)15692-4

26. Dzialowski I, Pexman JH, Barber PA, Demchuk AM, Buchan AM, Hill MD (2007) Asymptomatic hemorrhage after thrombolysis may not be benign: prognosis by hemorrhage type in the Canadian alteplase for stroke effectiveness study registry. Stroke 38(1):7579. doi:10.1161/01.STR.0000251644.76546.62

27. Park JH, Ko Y, Kim WJ, Jang MS, Yang MH, Han MK, Oh CW, Park SH, Lee J, Bae HJ, Gorelick PB (2012) Is asymptomatic hemorrhagic transformation really innocuous? Neurology 78(6):421426. doi:10.1212/WNL.0b013e318245d22c

28. Kimura K, Iguchi Y, Shibazaki K, Aoki J, Terasawa Y (2008) Hemorrhagic transformation of ischemic brain tissue after t-PA thrombolysis as detected by MRI may be asymptomatic, but impair neurological recovery. J Neurol Sci 272(1-2):136-142. doi:10. 1016/j.jns.2008.05.012

29. Saver JL (2006) Time is brain - quantified. Stroke 37(1):263-266. doi:10.1161/01.STR.0000196957.55928.ab

30. Tomsick T (2007) TIMI, TIBI, TICI: I came, I saw, I got confused. AJNR Am J Neuroradiol 28(2):382-384

31. Sanelli PC, Nicola G, Johnson R, Tsiouris AJ, Ougorets I, Knight C, Frommer B, Veronelli S, Zimmerman RD (2007) Effect of training and experience on qualitative and quantitative $\mathrm{CT}$ perfusion data. AJNR Am J Neuroradiol 28(3):428-432

32. Biesbroek JM, Niesten JM, Dankbaar JW, Biessels GJ, Velthuis BK, Reitsma JB, van der Schaaf IC (2013) Diagnostic accuracy 
of CT perfusion imaging for detecting acute ischemic stroke: a systematic review and meta-analysis. Cerebrovasc Dis 35(6):493501. doi:10.1159/000350200

33. van Seeters T, Biessels GJ, Niesten JM, van der Schaaf IC, Dankbaar JW, Horsch AD, Mali WP, Kappelle LJ, van der Graaf
Y, Velthuis BK (2013) Reliability of visual assessment of noncontrast CT, CT angiography source images and CT perfusion in patients with suspected ischemic stroke. PLoS One 8(10), e75615. doi:10.1371/journal.pone.0075615 